Miyuki Kobayashi, Kikuko lida, Hiroaki litsuka, Kun Li, Noriyuki Yonezawa and

Akiko Okamoto*

\title{
The crystal structure of
}

\section{1,8-bis(4-methoxybenzoyl)naphthalene-2,7-diyl dibenzoate, $\mathrm{C}_{40} \mathrm{H}_{28} \mathrm{O}_{8}$}

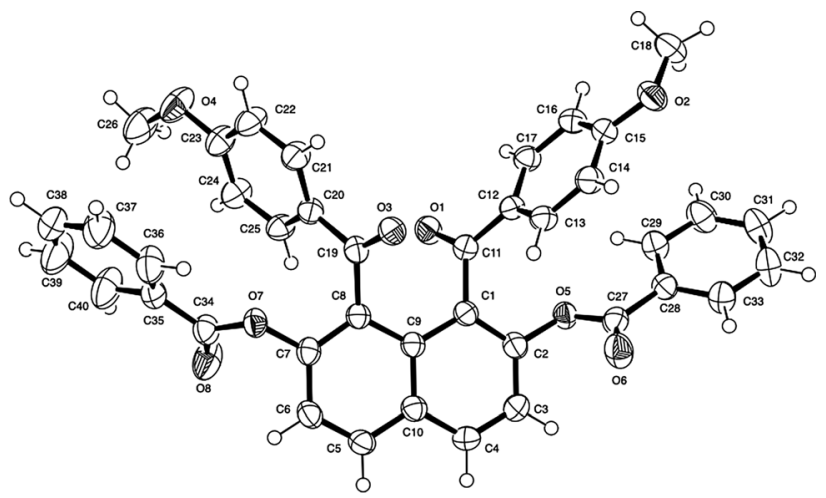

https://doi.org/10.1515/ncrs-2021-0160

Received April 27, 2021; accepted May 17, 2021;

published online June 23, 2021

\section{Abstract \\ $\mathrm{C}_{40} \mathrm{H}_{28} \mathrm{O}_{8}$, orthorhombic, $P_{n a 2}$ (no. 33), $a=27.0291(4) \AA$, $b=14.5593(2) \AA, c=7.95172(10) \AA, V=3129.20(7) \AA^{3}, Z=4$, $R_{g t}(F)=0.0276, w R_{r e f}\left(F^{2}\right)=0.0690, \mathrm{~T}=193 \mathrm{~K}$.}

CCDC no.: 2084108

The molecular structure is shown in the figure. Table 1 contains crystallographic data and Table 2 contains the list of the atoms including atomic coordinates and displacement parameters.

\section{Source of material}

The title compound was prepared by reaction of 1,8-bis (4-methoxybenzoyl)-2,7-dihydroxynaphthalene $(0.2 \mathrm{mmol}$,

*Corresponding author: Akiko Okamoto, Department of Organic and Polymer Materials Chemistry, Tokyo University of Agriculture and Technology, 2-24-16 Nakamachi, Koganei, Tokyo, Japan,

E-mail: aokamoto@cc.tuat.ac.jp. https://orcid.org/0000-0002-41480798

Miyuki Kobayashi, Kikuko lida, Hiroaki litsuka, Kun Li and Noriyuki Yonezawa, Department of Organic and Polymer Materials Chemistry, Tokyo University of Agriculture and Technology, 2-24-16 Nakamachi, Koganei, Tokyo, Japan
Table 1: Data collection and handling.

\begin{tabular}{|c|c|}
\hline Crystal: & Colorless block \\
\hline Size: & $0.40 \times 0.30 \times 0.10 \mathrm{~mm}$ \\
\hline Wavelength: & Cu $K \alpha$ radiation $(1.54187 \AA)$ \\
\hline$\mu$ : & $0.78 \mathrm{~mm}^{-1}$ \\
\hline Diffractometer, scan mode: & Rigaku R-AXIS RAPID, $\omega$ \\
\hline$\theta_{\max }$, completeness: & $68.2^{\circ},>99 \%$ \\
\hline$N(h k l)_{\text {measured }}, N(h k l)_{\text {unique }}, R_{\text {int }}:$ & $44,449,5717,0.032$ \\
\hline Criterion for $I_{\mathrm{obs}}, N\left(h k l_{\mathrm{gt}}\right.$ : & $I_{\text {obs }}>2 \sigma\left(I_{\text {obs }}\right), 5474$ \\
\hline$N(\text { param })_{\text {refined }}:$ & 436 \\
\hline Programs: & $\begin{array}{l}\text { Rigaku [1, 2], SIR2004 [3], SHELX } \\
\text { [4], ORTEP-III [5], PLATON [6] }\end{array}$ \\
\hline
\end{tabular}

$85.7 \mathrm{mg}$ ), which was obtained via $\mathrm{P}_{2} \mathrm{O}_{5}-\mathrm{MsOH}$ [7] -mediated diaroylation of 2,7-dihydroxynaphthalene and 4-methoxybenzoic acid, benzoyl chloride $(0.44 \mathrm{mmol}$, $61.9 \mathrm{mg})$, and triethylamine $(0.22 \mathrm{mmol}, 0.03 \mathrm{ml})$ in dichloromethane $(0.5 \mathrm{ml})$. After the reaction mixture was stirred at room temperature for $3 \mathrm{~h}$, it was poured into water $(30 \mathrm{ml})$ and the mixture was extracted with $\mathrm{CHCl}_{3}(10 \mathrm{ml} \times 3)$. The combined extracts were washed with sat. $\mathrm{NaHCO}_{3}$ aq. and brine. The organic layers thus obtained were dried over anhydrous $\mathrm{MgSO}_{4}$. The solvent was removed under reduced pressure to give the crude product (94\%). ${ }^{1} \mathbf{H}$ NMR $\delta$ $\left(400 \mathrm{MHz}, \mathrm{CDCl}_{3}\right): 3.73(6 \mathrm{H}, \mathrm{s}), 6.72(4 \mathrm{H}$, broad), 7.25 (4H, t, $J=6.4 \mathrm{~Hz}), 7.46(2 \mathrm{H}, \mathrm{t}, J=6.0 \mathrm{~Hz}), 7.56(2 \mathrm{H}, \mathrm{d}, J=7.6 \mathrm{~Hz}), 7.63$ ( $4 \mathrm{H}, \mathrm{d}, J=6.0 \mathrm{~Hz}), 7.69$ ( $4 \mathrm{H}$, broad), $8.10(2 \mathrm{H}, \mathrm{d}, J=7.6 \mathrm{~Hz})$ ppm ${ }^{13}$ C NMR $\delta$ (75 MHz, $\mathrm{CDCl}_{3}$ ): 55.45, 113.52 (overlap), 122.11, 128.31, 128.48, 129.68, 129.99, 130.82, 131.38, 131.54, 133.61, 147.54, 163.64, 164.18, 193.36 ppm. IR (KBr): 3450 $(\mathrm{C}-\mathrm{H}), 2838\left(\mathrm{O}-\mathrm{CH}_{3}\right), 1735(\mathrm{OC}=\mathrm{O}), 1653(\mathrm{C}=\mathrm{O}), 1599(\mathrm{Ar})$, 1507 (Ar) $\mathrm{cm}^{-1}$. HRMS $(\mathrm{m} / z):[M+\mathrm{H}]^{+}$calcd. for $\mathrm{C}_{40} \mathrm{H}_{29} \mathrm{O}_{8}$, 637.1863 , found, 637.1910 . m.p. $=184.6-185.0^{\circ} \mathrm{C}$.

\section{Experimental details}

All $\mathrm{H}$ atoms were found in a difference map and were subsequently refined as riding atoms, with $\mathrm{C}-\mathrm{H}=0.95$ (aromatic) and 0.98 (methyl) $\AA$, and with $U_{\text {iso }}(\mathrm{H})=1.2 U_{\text {eq }}(\mathrm{C})$. 
Table 2: Fractional atomic coordinates and isotropic or equivalent isotropic displacement parameters $\left(\AA^{2}\right)$.

\begin{tabular}{|c|c|c|c|c|}
\hline Atom & $x$ & $y$ & $z$ & $U_{\text {iso }} * / U_{\text {eq }}$ \\
\hline 1 & $0.29041(5)$ & $0.41345(10)$ & 0.86408 (19) & $0.0422(3)$ \\
\hline 02 & $0.13130(6)$ & $0.13796(11)$ & $1.1669(2)$ & 0.0505 (4) \\
\hline 03 & $0.26450(5)$ & $0.28654(9)$ & $0.5490(2)$ & 0.0403 \\
\hline 04 & $0.49626(6)$ & $0.24230(11)$ & $0.6109(3)$ & $0.0622(5)$ \\
\hline 05 & $0.16377(5)$ & $0.50203(10)$ & $0.92579(17)$ & $0.0367(3)$ \\
\hline 06 & $0.09082(6)$ & 0.46659 (13) & $0.8054(2)$ & 0.0544 (4) \\
\hline 07 & $0.33903(5)$ & $0.41496(9)$ & $0.24556(18)$ & $0.0368(3)$ \\
\hline 08 & $0.39186(6)$ & $0.53448(11)$ & $0.2487(3)$ & $0.0653(5)$ \\
\hline $\mathrm{C} 1$ & $0.22243(7)$ & $0.46811(13)$ & $0.7096(2)$ & $0.0323(4)$ \\
\hline $\mathrm{C} 2$ & $0.18339(7)$ & $0.52016(14)$ & $0.7655(3)$ & $0.0363(4)$ \\
\hline C3 & $0.16461(8)$ & 0.59593 (15) & $0.6767(3)$ & $0.0420(5)$ \\
\hline H3 & 0.137524 & 0.630192 & 0.719852 & $0.050^{*}$ \\
\hline $\mathrm{C} 4$ & $0.18584(8)$ & $0.61926(14)$ & $0.5282(3)$ & 0.0407 (5) \\
\hline H4 & 0.173861 & 0.671224 & 0.468495 & $0.049^{*}$ \\
\hline $\mathrm{C} 5$ & $0.24628(7)$ & $0.59382(14)$ & $0.3044(3)$ & .0384 (5) \\
\hline H5 & 0.233473 & 0.645536 & 0.245988 & $0.046^{*}$ \\
\hline C6 & $0.28432(7)$ & $0.54571(14)$ & $0.2374(3)$ & $0.0389(5)$ \\
\hline H6 & 0.298509 & 0.563885 & 133266 & $0.047^{\star}$ \\
\hline $\mathrm{C} 7$ & $0.30253(7)$ & $0.46888(13)$ & $0.3232(3)$ & 0.0344 (4) \\
\hline $\mathrm{C} 8$ & 0.28 & 0.43 & $2(3)$ & 19 (4) \\
\hline C9 & $0.24404(7)$ & $0.49011(12)$ & $0.5495(2)$ & 0.0314 (4) \\
\hline C10 & $0.22535(7)$ & 0.567 & $0.4607(3)$ & $50(4)$ \\
\hline 11 & $0.24682(7)$ & $0.40204(13)$ & $0.8329(3)$ & $31(4)$ \\
\hline 12 & $0.21717(7)$ & 0.33 & $0.9162(2)$ & $0.0332(4)$ \\
\hline 13 & $0.23196(7)$ & 0.29951 (13) & $1.0742(3)$ & $0.0372(4)$ \\
\hline $\mathrm{H} 13$ & 0.261607 & 0.322872 & 22056 & $0.045^{*}$ \\
\hline C14 & $0.20447(7)$ & $0.23586(15)$ & $30(3)$ & $.0401(5)$ \\
\hline H14 & 0.214743 & 0.216418 & 271590 & $0.048^{\star}$ \\
\hline C15 & $0.16175(7)$ & 0.20 & $15(3)$ & $3(5)$ \\
\hline 16 & $0.14703(8)$ & $0.22875(14)$ & $15(3)$ & $0.0392(5)$ \\
\hline H16 & 0.118268 & 0.203033 & 1516 & $0.047^{\star}$ \\
\hline 17 & $0.17416(7)$ & $0.29355(14)$ & $0.8461(3)$ & $.0360(4)$ \\
\hline H17 & 0.163532 & 3603 & 8288 & $0.043^{*}$ \\
\hline C18 & $.14346(10)$ & 0.10974 (18) & $1.3333(3)$ & $.0570(6)$ \\
\hline $18 \mathrm{~A}$ & 0.145922 & 3815 & 6193 & $0.068^{\star}$ \\
\hline $\mathrm{H} 18 \mathrm{~B}$ & 0.175225 & 0.077275 & 332191 & $0.068^{*}$ \\
\hline $\mathrm{H} 18 \mathrm{C}$ & 0.117622 & 0.068685 & 5994 & $0.068^{\star}$ \\
\hline C19 & $0.29736(7)$ & 0.34 & $353(2)$ & 0.0337 (4) \\
\hline $\mathrm{C} 20$ & $0.34972(7)$ & 0.32 & $68(3)$ & $0.0356(4)$ \\
\hline C21 & $0.36423(8)$ & 0.22857 (14) & $0.5707(3)$ & $0.0422(5)$ \\
\hline $\mathrm{H} 21$ & 0.339964 & 0.181619 & 0.561617 & $0.051^{*}$ \\
\hline $\mathrm{C} 22$ & $0.41302(8)$ & $0.20543(15)$ & $0.5874(4)$ & $0.0496(6)$ \\
\hline 22 & 0.422356 & 0.142538 & 0.589969 & $0.060^{*}$ \\
\hline $\mathrm{C} 23$ & $0.44902(8)$ & 0.27307 (16) & $0.6007(3)$ & $0.0470(5)$ \\
\hline C24 & $0.43507(8)$ & $0.36500(15)$ & $0.6039(3)$ & $0.0462(5)$ \\
\hline $\mathrm{H} 24$ & 0.459316 & 0.411667 & 0.617341 & $0.055^{*}$ \\
\hline $\mathrm{C} 25$ & $0.38586(7)$ & $0.38792(14)$ & $0.5873(3)$ & $0.0399(5)$ \\
\hline $\mathrm{H} 25$ & 0.376431 & 0.450767 & 0.589881 & $0.048^{*}$ \\
\hline $\mathrm{C} 26$ & $0.53508(9)$ & $0.3078(2)$ & $0.6011(5)$ & $0.0716(8)$ \\
\hline $26 \mathrm{~A}$ & 0.533231 & 0.349302 & 0.697866 & $0.086^{*}$ \\
\hline $\mathrm{H} 26 \mathrm{~B}$ & 0.531783 & 0.343284 & 0.496972 & $0.086^{*}$ \\
\hline $\mathrm{H} 26 \mathrm{C}$ & 0.567026 & 0.275940 & 0.601610 & $0.086^{*}$ \\
\hline $\mathrm{C} 27$ & $0.11558(8)$ & $0.47378(14)$ & $0.9297(3)$ & $0.0387(5)$ \\
\hline 28 & $0.09848(8)$ & $0.45404(13)$ & $1.1030(3)$ & $0.0362(4)$ \\
\hline C29 & $0.13081(8)$ & $0.44668(16)$ & $1.2368(3)$ & $0.0442(5)$ \\
\hline
\end{tabular}

\section{Comment}

Weak interactions in solid organic molecular substances have acquired growing interests and the consequent revaluation over the last two decades [8-10]. Since the degree of freedom in arranging aromatic rings is small, crystals of compounds of non-coplanarly accumulated aromatic rings can be expected to distinguish contributions of rather weak non-covalent bonding interactions other than $\pi \cdots \pi$ stacking interactions or classical hydrogen bonds as structure determining factors [11-13].

In the molecular structure of the title compound, the benzene rings of 1,8-(4-methoxybenzoyl) groups and those of 2,7-benzoyloxy groups are twisted away from the naphthalene ring (Figure). Two 1,8-(4-methoxybenzoyl) groups are situated in anti-orientation. The dihedral angles between the benzene rings of 4-methoxybenzoyl groups and the naphthalene ring system are $67.41(7)^{\circ}$ [C20-C25 ring, $\mathrm{C} 7-\mathrm{C} 8-\mathrm{C} 19-\mathrm{O} 3$ torsion angle $\left.=-116.81(15)^{\circ}\right]$ and $73.77(6)^{\circ}$ $\left[\mathrm{C} 12-\mathrm{C} 17\right.$ ring $\mathrm{C} 2-\mathrm{C} 1-\mathrm{C} 11-01$ torsion angle $\left.=-119.46(15)^{\circ}\right]$, respectively. The dihedral angle between the best planes of the two phenyl rings is $63.18(8)^{\circ}$. The two benzoyloxy groups at 2,7-positions of the naphthalene moiety are also situated in opposite directions. The dihedral angles between their benzene rings [ $\mathrm{C} 35-\mathrm{C} 40$ ring and $\mathrm{C} 28-\mathrm{C} 33$ ring] and the naphthalene ring system are $72.13(9)^{\circ}$ and $53.08(7)^{\circ}$, respectively. The two benzene rings make a dihedral angle of $77.55(10)^{\circ}$. The phenyl rings and carbonyloxy moieties are almost coplanar [08-C34-C35-C36 torsion angle $=-171.15(19)^{\circ}$ and $\mathrm{O} 6-\mathrm{C} 27-\mathrm{C} 28-\mathrm{C} 29$ torsion angle $\left.=167.41(16)^{\circ}\right]$. Several non-classical hydrogen bonds exhibiting almost similar interatomic distances are also confirmed: $\mathrm{C}-\mathrm{H}$ (methoxy) $\cdots \mathrm{O}=\mathrm{C}$ (benzoyl) $(2.57 \AA$, H18B $\cdots 01 ; 1 / 2-x,-1 / 2+y, 1 / 2+z), \mathrm{C}-\mathrm{H}($ naphthalene) $\cdots$ $\mathrm{O}=\mathrm{C}$ (benzoyl) $(2.58 \AA$, H5 $\cdots 03 ; 1 / 2-x,-1 / 2+y, 1 / 2+z)$,

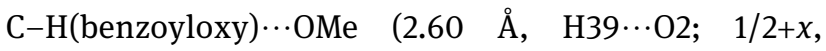
$1 / 2-y,-1+z$ ), and and $\mathrm{C}-\mathrm{H}$ (benzoyloxy) $\cdots \mathrm{O}$ (benzoyloxy)

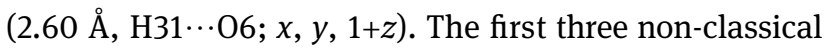
hydrogen bonds are parallel to the glide planes, and the last one is oriented along the $\mathrm{c}$ axis. The authors reported the crystal structure of the homologous compounds [2,7-dimethoxy-8-(4-methoxybenzoyl)-naphthalen1-yl](4-methoxyphenyl)methanone chloroform monosolvate (I) [14] and 1,8-dibenzoylnaphthalene-2,7-diyl dibenzoate (II) [15]. The title compound and the homologue II shows unsymmetric spatial situation of 1,8-diaroyl groups on naphthalene ring [dihedral angles between phenyl rings and naphthalene: $67.41(7)^{\circ}$ and $73.77(6)^{\circ}$ for the title compound, $67.12(5)^{\circ}$ and $85.15(5)^{\circ}$ for homologue II, and $72.51(7)^{\circ}$ and $73.33(7)^{\circ}$ for homologue I]. 
Table 2: (continued)

\begin{tabular}{lrrrr}
\hline Atom & $\boldsymbol{x}$ & $\boldsymbol{y}$ & $\boldsymbol{z}$ & $\boldsymbol{U}_{\text {iso }}{ }^{*} \boldsymbol{U}_{\text {eq }}$ \\
\hline H29 & 0.165393 & 0.453749 & 1.219787 & $0.053^{*}$ \\
C30 & $0.11237(10)$ & $0.42890(18)$ & $1.3959(3)$ & $0.0527(6)$ \\
H30 & 0.134439 & 0.423525 & 1.488397 & $0.063^{*}$ \\
C31 & $0.06230(10)$ & $0.41897(18)$ & $1.4210(3)$ & $0.0564(6)$ \\
H31 & 0.049884 & 0.407391 & 1.530749 & $0.068^{\star}$ \\
C32 & $0.03018(9)$ & $0.4258(2)$ & $1.2874(3)$ & $0.0565(6)$ \\
H32 & -0.004362 & 0.418441 & 1.304900 & $0.068^{\star}$ \\
C33 & $0.04805(8)$ & $0.44328(16)$ & $1.1281(3)$ & $0.0455(5)$ \\
H33 & 0.025869 & 0.447931 & 1.035841 & $0.055^{*}$ \\
C34 & $0.38375(8)$ & $0.45543(15)$ & $0.2158(3)$ & $0.0423(5)$ \\
C35 & $0.42021(8)$ & $0.39016(15)$ & $0.1459(3)$ & $0.0431(5)$ \\
C36 & $0.40780(9)$ & $0.30444(16)$ & $0.0899(4)$ & $0.0560(6)$ \\
H36 & 0.374231 & 0.285137 & 0.091985 & $0.067^{*}$ \\
C37 & $0.44404(11)$ & $0.24566(19)$ & $0.0301(4)$ & $0.0706(8)$ \\
H37 & 0.435204 & 0.186238 & -0.009013 & $0.085^{*}$ \\
C38 & $0.49228(11)$ & $0.2726(2)$ & $0.0272(4)$ & $0.0666(8)$ \\
H38 & 0.516992 & 0.231959 & -0.013693 & $0.080^{*}$ \\
C39 & $0.50526(10)$ & $0.3585(2)$ & $0.0833(5)$ & $0.0781(9)$ \\
H39 & 0.538913 & 0.377240 & 0.081522 & $0.094^{*}$ \\
C40 & $0.46926(9)$ & $0.4174(2)$ & $0.1423(5)$ & $0.0709(9)$ \\
H40 & 0.478170 & 0.476900 & 0.180721 & $0.085^{*}$ \\
\hline
\end{tabular}

Two benzoyloxy groups in homologue II are twisted to the naphthalene ring with almost same dihedral angles $\left[71.47(5)^{\circ}\right.$ and $76.41(5)^{\circ}$ vs. $72.13(9)^{\circ}$ and $53.08(7)^{\circ}$ for the title compound]. In these compounds, fewer non-classical hydrogen bonds than in the packing of the title compound are observed, e.g. $\mathrm{C}-\mathrm{H}$ (naphthalene) $\cdots$ OMe for homologue I, $\mathrm{C}-\mathrm{H}$ (benzoyl) $\cdots \mathrm{O}=\mathrm{C}$ (benzoyl) and $\mathrm{C}-\mathrm{H}$ (benzoyloxy) $\cdots \pi$ (naphthalene) for homologue II. More effective factors stabilize their crystals such as co-crystallization with solvent molecules or $\pi \cdots \pi$ stacking interactions. In other words, continuous neighbouring substitution of 4-methoxybenzoyl groups and benzoyloxy group disturb the effective interactions other than non-classical hydrogen bonds in title compound. Concludingly, neighbourly aligned four benzene rings on naphthalene ring afford enough stabilization with the aid of maximum number of non-classical hydrogen bonds in the absence of $\pi \cdots \pi$ stacking. The magnitude of the stabilization satisfactorily compensates those expected by intermolecular interaction of crystal solvent.

Acknowledgement: The authors express their gratitude to Professor Keiichi Noguchi, Instrumentation Analysis Center, Tokyo University of Agriculture and Technology, for technical advice.
Author contributions: All the authors have accepted responsibility for the entire content of this submitted manuscript and approved submission.

Research funding: JSPS KAKENHI Grant Number JP20K05473. Tokyo Ohka Foundation for The Promotion of Science and Technology Grant Number 216065.

Conflict of interest statement: The authors declare no conflicts of interest regarding this article.

\section{References}

1. Rigaku. PROCESS-AUTO; Rigaku Corporation: Tokyo, Japan, 1998.

2. Rigaku. CRYSTALSTRUCTURE; Rigaku Corporation: Tokyo, Japan, 2010.

3. Burla M. C., Caliandro R., Camalli M., Carrozzini B., Cascarano G. L., De Caro L., Giacovazzo C., Polidori G., Spagna R. SIR2004: an improved tool for crystal structure determination and refinement. J. Appl. Crystallogr. 2005, 38, 381-388.

4. Sheldrick G. M. A short history of SHELX. Acta Crystallogr. 2008, A64, 112-122.

5. Burnett M. N., Johnson C. K. ORTEPIII. Report ORNL-6895; Oak Ridgeational Laboratory: Tennessee, USA, 1996.

6. Spek A. L. Structure validation in chemical crystallography. Acta Crystallogr. 2009, D65, 148-155.

7. Eaton P., Carlson G. R., Lee J. T. Phosphorus pentoxide-methane sulfonic acid. convenient alternative to polyphosphoric acid. J. Org. Chem. 1973, 38, 4071-4073.

8. Desiraju G. R., Steiner T. The Weak Hydrogen Bond in Structural Chemistry and Biology; Oxford University Press: Oxford, 1999.

9. Desiraju G. R. C-H $\cdots O$ and other weak hydrogen bonds. from crystal engineering to virtual screening. Chem. Commun. 2005, 24, 2995-3001.

10. Taylor R., Kennard O. Crystallographic evidence for the existence of $\mathrm{C}-\mathrm{H} \cdots \mathrm{O}, \mathrm{C}-\mathrm{H} \cdots \mathrm{N}$, and $\mathrm{C}-\mathrm{H} \cdots \mathrm{Cl}$ hydrogen bonds. J. Am. Chem. Soc. 1982, 104, 5063-5070.

11. Okamoto A., Yonezawa N. Unique and specific reaction behavior and characteristic spatial organization of non-coplanarly aromatic-ring-accumulated molecular compounds. J. Synth. Org. Chem. 2015, 73, 339-360.

12. Muto T., lida K., Noguchi K., Yonezawa N., Okamoto A. Crystal structure and Hirshfeld surface analysis of 2-hydroxy-7-methoxy1,8-bis(2,4,6-trichlorobenzoyl)naphthalene. Acta Crystallogr. 2019, E75, 1418-1422.

13. litsuka H., Li K., Kobayashi M., lida K., Yonezawa N., Okamoto A. Crystal structure of 1,2-bis(4-fluorophenyl)-1-hydroxy2,3,8-trimethoxyacenaphthene: formation of a five-membered intramolecular $\mathrm{O}-\mathrm{H} \cdots \mathrm{O}$ hydrogen-bonded ring. Acta Crystallogr. 2021, E77, 175-179.

14. Sasagawa K., Sakamoto R., Kusakabe T., Okamoto A., Yonezawa N. [2,7-Dimethoxy-8-(4-methoxybenzoyl)nephthalen-

1-yl](4-methoxyphenyl)methanone chloroform monosolvate. Acta Crystallogr. 2013, E69, 0146.

15. Sakamoto R., Sasagawa K., Hijikata D., Okamoto A., Yonezawa N. 1,8-Dibenzoylnaphthalene-2,7-diyl dibenzoate. Acta Crystallogr. 2012, E68, 02454. 